\title{
A Physiological Foundation for the Nutrition-based Efficiency Wage Model
}

\author{
Carl-Johan Dalgaard* and Holger Strulik ${ }^{* *}$ \\ Leibniz Universität Hannover, Discussion Paper No. 396 \\ ISSN 0949-9962
}

This Version: September 2010 (First Version: April 2008)

\begin{abstract}
Drawing on recent research on allometric scaling and energy consumption, the present paper develops a nutrition-based efficiency wage model from first principles. The biologically micro-founded model allows us to address empirical criticism of the original nutrition-based efficiency wage model. By extending the model with respect to heterogeneity in worker body size and a physiologically founded impact of body size on productivity, we demonstrate that the nutrition-based efficiency wage model is compatible with the empirical regularity that taller workers simultaneously earn higher wages and are less likely to be unemployed in less developed economies. The theory also provides an answer to the question of why the height-unemployment association may disappear in the process of development.
\end{abstract}

Keywords: Efficiency Wages, Nutrition, Metabolism, Allometric Scaling, Body Size.

JEL: O11, O15, J21, J31.

\footnotetext{
${ }^{*}$ University of Copenhagen, Department of Economics, Studiestraede 6, 1455 Copenhagen K, Denmark; email: carl.johan.dalgaard@econ.ku.dk.

**University of Hannover, Wirtschaftswissenschaftliche Fakultät, Königsworther Platz 1, 30167 Hannover, Germany; email: strulik@vwl.uni-hannover.de.
} 


\section{INTRODUCTION}

One of the most prominent theories of wage formation and unemployment in development economics is the nutrition based efficiency wage model due to Leibenstein (1957), Mirrlees (1976), Stiglitz (1976) and Bliss and Stern (1978a,b). The basic theory works as follows. Assume output is a concave function of labour input; the number of people and their effort level. Next, suppose higher wages allow for a higher nutritional intake of workers, which stimulates effort. Then the optimal wage, from the perspective of the producer, will be the one which minimises the wage bill in efficiency units of labour (i.e., the wage rate divided by the effort level). If one makes the appropriate assumptions about the nature of the effort function, linking effort to wages, an interior solution exists. Figure 1 provides a geometric illustration; $w^{*}$ is thus the wage level which minimises labour cost. Given the wage, thus determined, the level of employment is given by the first order condition from profit maximisation: the marginal product of labour equals its factor price. Insofar as total employment, obtained by summing across firms, falls short of total labour supply, unemployment arises in equilibrium. Moreover, since the wage is optimal from the perspective of employers, unemployed laborers cannot undercut.

Figure 1: Determination of the Efficiency Wage

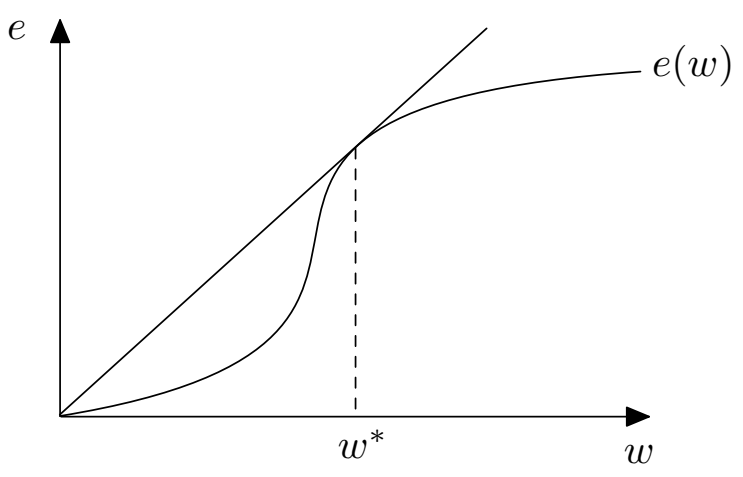

Albeit highly influential, the nutrition-based efficiency wage theory has been criticised empirically. While in theory the level of wages is essentially given by the curvature of the effort function, which is unobserved and unspecified by the theory, one may nevertheless attempt a test of the model by examining whether wages responds to the nutritional intake of workers (calories per day, say). Such a link should be present in the data, although this test is only a 
test of the reduced form of the model. ${ }^{1}$ Some past research has established a positive impact of calorie intake on wages (Strauss, 1986). Unfortunately, however, this association seems to disappear once the body size of workers is taken into account (Deolalikar, 1988). Theoretically, worker body size could matter to wages independently of effort. This would be the case if body size proxies the health of workers, which in turn affects the marginal product of labour and thus wages (e.g, Fogel, 1994; Schultz, 2002; Weil, 2007).

As a result, the findings of Deolalikar (1988) are troubling; they suggest the nutrition-wage link mainly is spurious, possibly reflecting the omission of health from the production function, and that richer (healthier) workers spend more on nutrition. Accordingly, such findings instil doubt as to whether the nutrition-based theory of wage determination is important in practice.

In this paper we address this critique theoretically by providing a physiological foundation for the effort function with strong micro-foundations. The theory provides a more precise definition of (i) the notion of effort, and (ii) how energy requirements (and therefore wage requirements) depend on effort. We base these micro-foundations on recent research in the fields of biology and human nutrition.

On this basis we solve for the partial equilibrium in the labour market, demonstrating the viability of the unemployment equilibrium. In addition, we derive equilibrium wages, as a function of worker characteristics, and proceed to show that the micro-founded theory predicts that there should be no association between wages and nutrition, conditional on body size. This resolves the apparent tension between available evidence and the efficiency wage hypothesis. That is, it explains why findings of e.g. Deolalikar, (1988) do not falsify the nutrition-based efficiency wage model. At the same time, developing micro-foundations for the effort function provides important clues as to how stronger tests of the model may be devised.

Another benefit of providing micro-foundations for the effort function is that we can calibrate the model. As a result, we can quantify the implied association between body size of workers and wages. In addition, we can assess the size of the gap between equilibrium wages, and the hypothetical wage needed to cover energy expenditures for subsistence. ${ }^{2}$ This allows us to address a second line of criticism marshalled against the nutrition-based efficiency wage model.

\footnotetext{
${ }^{1}$ The test would fail if either nutrition does not raise effort (suitably defined), or if additional effort does not raise wages.

${ }^{2}$ In this paper we follow Dalgaard and Strulik (2010) in defining the level of subsistence as the amount of energy required to cover basal metabolic needs. Metabolism refers to the biochemical processes by which nutrients are transformed into energy, which allows the organs of the body (i.e. ultimately the cells of the body) to function. The basal metabolic rate is defined as the amount of energy expended while at rest.
} 
Existing empirical work has demonstrated that wages in poor places seem to be (far) above subsistence. For example, Dasgupta (1997) notes (p. 31) that in India 15-20\% of wages paid should be enough to cover personal energy requirements thus suggesting an $80 \%$ wage premium. At first sight, wages way above subsistence seem hard to reconcile with the efficiency wage model, as one might imagine that workers cannot be energy constrained in this region. As a result, one may argue that undercutting should arise (see Swamy, 1997). The baseline model can, however, under reasonable (i.e., biologically supported) assumptions easily account for an $80 \%$ wage premium. Hence, wages considerably above subsistence need not instigate undercutting.

Physical activity is not the only reason why average wages exceed the subsistence threshold by a considerable margin, as made clear in an extension of the basic model. In the baseline model we employ the notion of a representative worker, of given body size. In practice, of course, people come in different sizes. We therefore extend the model by allowing for heterogeneity in stature, so as to examine which workers will be rationed in equilibrium.

In the extended model we specify how body size matters to productivity, for effort given. Drawing on research in sports physiology and biology, we explain how stature should relate to physical performance, and thus productivity. This aspect of the model is based on the idea that many occupations in poor economies are of a manual variety. Much like body size importantly influences the performance of athletes in various disciplines, we argue the same would be true for worker performance in firms. The nature of the link between body size and performance, however, critically depends on the specific task the worker is assumed to perform. Consequently we can characterise situations where the augmented efficiency wage model would lead us to expect that larger or smaller workers are rationed.

In the case where large workers are preferred, and we would argue it is the typical outcome in poor economies, selection will imply that standard calculations of the wage gap, mentioned above, may be misleading. The reason is that personal energy requirements typically refer to that of an average individual in the labour force. But if in equilibrium only larger people tend to be employed (so that the average person employed is larger than the average in the labour force), part of what would seem to be a wage premium may reflect the positive selection of workers with larger energy requirements than the average. ${ }^{3}$

\footnotetext{
${ }^{3}$ Strauss and Thomas (1998) provide evidence that smaller individuals, in poor countries, are less likely to be working.
} 
Hence, the augmented model provides a second reason why small and 'cheap' workers cannot undercut wages. While smaller people may be cheaper to sustain, they are not attractive to firms since they are less productive than larger and more energy costly individuals. ${ }^{4}$

The model featuring size heterogeneity also allows us to discuss the concept of task-specific technological innovations. Below, we hypothesise that technology usually reduces energy requirements of humans in the production process. This process may manifest itself in changing the nature of the selection process of workers, as the nature of the work done at the level of firm's changes. If, for example, technological change reduces the need for 'brawn', smaller workers (e.g., women and perhaps children) will become more attractive to employers. ${ }^{5}$ Finally, we examine the efficiency wage model in the case where effort is imperfectly observable. In this case wages may be set sub-optimally low by the employer, based on his or her expectation about worker effort. Nevertheless undercutting will not take place. A new interesting result from this extension is that employers are less likely to select in favour of physically large workers when work is done at a piece-rate basis.

The precursors to the present paper are the theoretical contributions to the literature on efficiency wages: Mirrlees (1976), Stiglitz (1976), Bliss and Stern (1978a,b), Dasgupta and Ray (1986) and Bose (1997). Also related is the model of nutrition and productivity by Svedberg (2000, Ch. 3). Bliss and Stern (1978b) is of particular relevance since the authors discuss the nutritional basis for the efficiency wage hypothesis.

The present paper has, however, the advantage of being able to draw on more recent research in biology and from the science of nutrition. As a result our approach differs in a number of important respects. First, we employ a different conceptualisation of effort. Whereas Bliss and Stern (1978b) conceptualise effort as the number of 'tasks' performed (during a day, say), we associate effort with the physical activity level (PAL) in keeping with research in human nutrition and physiology. The key advantage of employing PAL in characterizing effort is that it is an appropriate metric for evaluating the intensity of work in any occupation. In contrast, a 'task' can involve greatly varying degrees of work intensity depending on how it is defined.

Second, more recent research in biology leads us to a different specification of the energy costs of such effort, compared to what Bliss and Stern explores. Finally, Bliss and Stern (1978b) do

\footnotetext{
${ }^{4}$ Yet another reason for lack of undercutting could be that the individual worker may have a family to support, thus preventing undercutting even if wages exceed personal energy expenditure (Dasgupta, 1997).

${ }^{5}$ This idea is related to, but distinct from, the notion that capital accumulation increases the return to 'brains' relative to 'brawn' (Galor and Weil, 1996).
} 
not discuss the physiological influence of body size on labour productivity, and do not consider heterogeneity with respect to body size in their formal analysis.

The paper is structured as follows. The next section develops the baseline model. Section 3 then augments the model, by allowing physiology to directly influence labour productivity, and by allowing for heterogeneity in body size. Section 4 investigates how results change if wages are sub-optimally low due to imperfectly observable effort. A final section concludes.

\section{THE BASIC MODEL}

2.1. A biological foundation for the effort function. The first step in deriving the effort function is to formalise minimum consumption requirements; food requirements while at rest. In this endeavour we invoke Kleiber's law which states that the basal metabolic rate of an organism $E_{0}$, measured by calories per day, scales with the mass of an organism $m$ as $E_{0}=a \cdot m^{b}$ with $b=3 / 4$ (Kleiber, 1932). Intuitively the law says that bigger organisms are more efficient; they need to consume less energy per unit of mass (i.e. per cell if the single cell is an mass invariant unit) in order to sustain their life. The 3/4 exponent has been verified by Brody (1945) for almost all terrestrial animals yielding the famous mouse-to-elephant curve. Although the law has long been known, it is only recently that teams of biologists and physicists have developed a theory which shows that the $3 / 4$ exponent follows from nature's optimisation of fluid flows through energy distributing networks like, for example, blood vessels. Accordingly, the formula $E_{0}=a \cdot m^{b}$ can be given rather deep micro-foundations (West et al., 1997; Banavar et al.,1999). ${ }^{6}$ Since basal metabolism is defined as energy needs of a body at rest, it is a useful concept to describe the nutritional needs of a worker who is exerting no effort at all like, for example, somebody who is lying in the shade all day.

Exerting effort, however, requires additional energy intake in order to support the muscular contractions involved in body postures and movements. Empirically it has been documented that both the proportionality constant $a$ and the scaling exponent $b$ rise for exercising animals and humans. Studies by Leonard and Robertson (1992), Darveau et al. (2002), White and Seymour (2005), Weibel et al. (2004) and Weibel and Hoppeler (2005) find values for the exponent between 0.82 and 0.92 , depending on the organism and the task under investigation.

\footnotetext{
${ }^{6}$ By now the theory has been applied to a multitude of biological problems from 'genomes to ecosystems' (West and Brown, 2005). In Dalgaard and Strulik (2010) we provide a brief introduction to the energy network theory and a first economic application on the development of human body size and population size over the long-run.
} 
Recently, da Silva et al. (2007) have generalised the theory of energy distributing networks to the case of moving organisms, suggesting a scaling exponent of $6 / 7$ for maximum metabolism.

The energy needs of an active body admit a biologically founded conceptualisation of effort. Let effort $e$ represent a measure of the extent of physical activity per day, and normalize such that $e$ falls in a $(0,1)$ interval. Accordingly, $e=0$ means no physical activity during the day, whereas $e=1$ denotes the maximum level of physical activity per day. In contrast to Bliss and Stern $(1976 \mathrm{a}, \mathrm{b})$ this notion of "activity" is not to be viewed as a measure of the number of "tasks" being performed. Instead we show that there is a close correspondence between $e$ and what nutritionists and physiologists refer to as "the physical activity level" (PAL). ${ }^{7}$ Indeed, as $e$ rises, in our notation, PAL will rise as well. As explained below, PAL cannot increase arbitrarily, but is constrained by physiology. This fact will eventually allow us to calibrate key biological parameters of the model.

The association between effort and energy requirements is now obtained by observing that energy intake of an active body can be written as the product of basal metabolism and extra energy needs for activity, i.e. $E \propto m^{b} \cdot m^{c}$ with $b=3 / 4$, and $c=(6 / 7)-(3 / 4)=3 / 28$ according to da Silva et al.'s theory. Thus, for computing energy expenditure of a body exerting effort $e$ per day and being inactive the remainder of the day the weighted geometric mean constitutes the appropriate measure. As a result, energy requirements are obtained as $E(e)=\left(a m^{b}\right)^{1-e}$. $\left(a_{e} m^{b+c}\right)^{e}$ where $a_{e}$ denotes the proportionality constant when $e=1$; at maximum daily activity level the organism reaches maximum metabolism, $a_{e} m^{b+c}$, whereas complete inactivity implies basal metabolism in keeping with Kleiber's law, $a \cdot m^{b}$. A virtue of this formulation is that experimental data on humans reveal that total energy expenditure rises with physical activity $e$ in a manner consistent with the $m^{b+e \cdot c}$ formula (see Westerterp, 2001 and the discussion below). The assumption that energy needs rise with the degree of physical activity is thus supported by theory and evidence deriving from biology and by work done by nutritionists. This is the centerpiece of the biologically founded effort function.

To complete the description of energy needs associated with effort we need some additional notation. Let $\eta$ be the ratio of the constants of proportionality with and without effort, $\eta \equiv$ $a_{e} / a \geq 1$. Total energy needed to sustain the metabolism of a worker of mass (weight) $m$ and effort level $e$ can then by simplified to $a \eta^{e} m^{b+e \cdot c}$. Let $\epsilon$ denote an 'energy exchange rate'

${ }^{7} \mathrm{PAL}$ is defined as total energy expenditure (per unit of time) divided by the Basal Metabolic Rate (per unit of time). See Food and Agriculture Organization of the United Nations (FAO, 2001, p. 16). 
that converts consumption in units of goods (wage income) into consumption in units of energy (calories). ${ }^{8}$ The wage which is sufficient to cover energy needs at effort level $e$ is:

$$
w(e) \cdot \epsilon=a \eta^{e} m^{b+e \cdot c} .
$$

Solving (1) for effort we get

$$
e=\frac{\log (w)-\log (a)+\log (\epsilon)-b \log (m)}{\log (\eta)+c \log (m)} \equiv e(w) .
$$

This is the effort function which we will employ below.

Here we consider a static model where we require workers to be compensated fully for their work effort in accordance with equation (2). Full compensation of effort can also be conceptualised as the long-run steady-state solution of a dynamic model. In a dynamic framework, body weight would be an accumulable state variable and employers could feed up emaciated job candidates by paying wages above metabolic needs at full effort level. For this to occur as a matter of transitional dynamics, enforceable long-term contracts are required; this case is investigated by Bose (1997). Feeding up casual workers would clearly be suboptimal. Given long-term contracts it is also conceivable that already hired workers receive wages below their energy needs for a while and nevertheless continue to exert effort. This could arise during a period of economic hardship, because the employer credibly commits to feed up the worker once the economy recovers. ${ }^{9}$ But in a steady-state, which is what we investigate here, effort needs to be fully compensated in a market economy in keeping with equation (2).

2.2. Analysis. Consider the labour demand and wage compensation problem of a firm facing a homogenous labour supply and a neoclassical production function

$$
Y=F(e(w) \cdot L)
$$

with $F^{\prime}>0$ and $F^{\prime \prime}<0$. Here $L$ is the number of employees and $e$ is the effort of an employee. Effort depends on the wage paid; $e \in(0,1)$ where 0 denotes no effort and 1 maximum effort. The first order conditions for maximizing output minus labour costs, $Y-w L$, with respect to

\footnotetext{
${ }^{8}$ This will later serve as a useful tool in analyzing the impact of a change in diet, or agricultural progress, as a comparative static exercise.

${ }^{9}$ Otherwise, without commitment, in a market solution it is impossible that the worker exerts effort given wages below energy needs. He would just quit and stop working rather than losing his life. However, beyond the market solution one can imagine a forced labour scenario where the employer is paying wages below energy requirements yet forces the worker to exert full effort. We do not consider this possibility in the present paper.
} 
$w$ and $L$ leads to the familiar Solow (1979) condition

$$
\frac{\partial e}{\partial w} \cdot \frac{w}{e}=1
$$

According to the nutrition-based theory of efficiency wages we assume that wage income is used for consumption of food and that effort at work depends on the individual level of nutrition. In contrast to the earlier work, however, we anchor the nutrition-effort mechanism in biological fundamentals, as described above.

Taking the first derivative of equation (2) and employing the Solow condition (4) we obtain the optimal wage $w=\exp (1) \cdot(a \epsilon) \cdot m^{b}$. Substituting the result back into (2) yields the associated effort level $e=1 /[\log (\eta)+c \cdot \log (m)]$ as interior solution. If $\log (m)<[1-\log (\eta)] / c$, a corner solution applies where workers exercise full effort $(e=1)$ and metabolic needs according to (1) imply wages of $\left(a_{e} / \epsilon\right) \cdot m^{b+c}$. The complete solution of the efficiency wage problem thus reads

$$
\begin{aligned}
& e^{*}=\min \left\{1, \frac{1}{\log (\eta)+c \cdot \log (m)}\right\} \\
& w^{*}=\min \left\{\frac{a \eta}{\epsilon} \cdot m^{b+c}, \exp (1) \cdot \frac{a}{\epsilon} \cdot m^{b}\right\} .
\end{aligned}
$$

In order to check whether the solution is interior we solve for the critical $m$ for which the corner solution is just binding, $m_{c r i t}=\exp [(1-\log \eta) / c]$. If, for example, $\eta=1.6$ and $c=6 / 7$, the critical weight is $140 \mathrm{~kg}$. Using a calibration of the model we will argue below that for physiological reasons $\eta$ is very likely to be below $1.6 ; m_{\text {crit }}=140 \mathrm{~kg}$ is therefore likely to be a lower bound. The corner solution is thus the empirically relevant one.

This result is of interest because it refutes the assumption of the standard efficiency wage model (e.g. Mirrlees, 1976) that profit maximizing employers face a convex-concave effort function and set wages that support an interior solution, i.e. less than maximum effort. At the same time our result continues to abide by the main premise of the standard efficiency wage model: better nutrition leads to higher effort.

Another important implication concerns equilibrium wages; the solution leaves no separate role for nutrition, conditional on the body mass of the worker. Hence the findings of Deolalikar (1988), which demonstrate that there is no association between wages and calorie intake once the stature of the worker is controlled for in the regression, is consistent with the model. Moreover, the estimates reported by Deolalikar (1988) are also quantitatively consistent with the present 
model. Using fixed effects Deolalikar (1988, Table 1) estimates a wage elasticity with respect to weight-for-height of 0.66 , evaluated at the mean of his sample. Allowing the point estimate to move 2 standard deviations to either side, implies a $95 \%$ confidence interval for the elasticity of $(0.13,1.2)$, which nests the prediction of the present model $(0.82,0.92)$.

Because the wage is pinned down by biological fundamentals, it determines employment (where $F^{\prime}\left(L^{*}\right)=w^{*}$ since $e=1$ in equilibrium) and consequently, as in the standard efficiency wage model, unemployment arises if the labour force exceeds $L^{*}$.

2.3. Quantitative issues. For the calibration of our model we begin with Kleiber's original formula for basal metabolism, i.e. $a=70, b=0.75$, in order to obtain energy needs for workers at rest $(e=0)$. Kleiber obtained these parameters for a sample of mammalian species but later it has been confirmed that they are not significantly different for a more narrow data set of 20 anthropoid species (Leonard and Robertson, 1992). ${ }^{10}$ As argued above the scaling exponent increases under activity, i.e. when workers exert effort. For a discussion of energy needs in activity we refer to a concept used by nutritionists, the physical activity level (PAL). The PAL has a clear correspondence in our model since it is defined as the ratio between energy needs in activity to energy needs at rest: $P A L(e)=\left(a \eta^{e} m^{b+e c}\right) /\left(a m^{b}\right)=\eta^{e} m^{e c}$.

According to the nutritional literature (FAO, 2001, Westerterp, 2001) humans cannot persistently sustain a PAL above 2.4 for extended periods of time. That is, a PAL of 2.4 can be considered the maximum maximorum (the unconditional upper bound). Naturally, during peak activity a much higher PAL can be reached temporarily. For example, activities like 'loading sacks on a truck' and 'carrying wood' are associated with PAL values of 6.6 (FAO, 2001) implying that a worker's energy needs would be 6.6 times his basal metabolic rate if he were occupied with these activities for 24 hours. At this level of physical labor, however, fatigue will set in, forcing the individual to rest. The periods of rest will automatically work so as to lower the average PAL for the day. For example, if the worker is constrained by a PAL of 2.4, this implies that he can only manage to exert effort in activities such as 'carrying wood' for about $2.4 \cdot 24 / 6.6=8.7$ hours per day (if we assume - in line with our model - that he exerts no effort at all over the rest of the day). Less energy consuming activities are sustainable for longer hours.

\footnotetext{
${ }^{10}$ Recently, it has been shown that for a sample of sedentary western humans, Kleiber's law can only be confirmed when one controls for obesity and age (Heymsfield et al. (2007). These characteristics play, however, no substantial role in our model focusing on workers in developing countries so that we continue with the original formula.
} 
Given the premise that the maximum (daily) sustainable PAL provides the nutritionist's equivalent to maximum metabolic effort in our model, we can calibrate the biological parameter $\eta$. In order to do so we proceed in a few simple steps.

To begin, we observe that an upper boundary for PAL at 2.4 implies, in theory, that $2.4=$ $\eta m_{\max }^{c}$, where $m_{\max }$ reflects (an estimate of) maximum human body mass; since PAL inevitably depends on body size, the upper boundary for metabolic effort should intuitively be associated with maximum body size. In order to pin down $m_{\max }$, we observe that the tallest man in recorded history is Robert Wadlow (born 1918) who stood at $2.72 \mathrm{~cm}$ at the time of his death in 1940 (McFarlan and McWhirter, 1991, p. 5). To get an implied body mass we invoke the body mass index (BMI). ${ }^{11}$ Assuming a BMI at the midpoint of the normal range (i.e. 21.75) leaves us with a reasonable guess for maximum (non-obese) body mass: $m_{\max }=2.72^{2} \cdot 21.75=160 \mathrm{~kg} .{ }^{12}$ Finally, we take the parameter $c$ from the biological literature; in theory $c=6 / 7-3 / 4=3 / 28$. As a result we obtain $\eta=2.4 / 160^{3 / 28}=1.39$. This is the calibrated $\eta$, which plausibly is consistent with the physiological upper boundary for PAL.

Before we present our calibrations for energy requirements in activity, we can invoke our calibrated $\eta$ to check the viability of the corner solution we derived above. With $\eta$ at 1.39 , we find that the corner solution applies as long as $m<m_{\text {crit }}=523 \mathrm{~kg}$; individuals lighter than half a ton, will exert full effort. This shows that the corner solution indeed is the empirically meaningful solution, under plausible biological parameter values. ${ }^{13}$

With a fixed calibrated value for $\eta$ it inevitably follows that our model admits minor variation in PAL across individuals of different body sizes. To illustrate, the right hand side panel of Figure 2 show the association between the physical activity level per day (i.e., "effort") and PAL for individuals at $65 \mathrm{~kg}$ (solid line), $90 \mathrm{~kg}$ (dashed), and $160 \mathrm{~kg}$ (dotted).

The first thing to notice from the figure is that, consistent with available evidence, PAL rises (almost) linearly with the degree of physical activity per day. Second, PAL falls in a 1 to 2.4 range. Both of these aspects are consistent with evidence (Westerterp, 2001; FAO, 2001). Third,

\footnotetext{
${ }^{11}$ The body mass index is defined as the ratio between the weight of an individual in $\mathrm{kg}$ relative to the individuals height squared, in meters.

${ }^{12}$ For obese persons there would be different PALs. As already mentioned we exclude this case by focusing on physically active workers in developing countries. Mr. Wadlow may have suffered from gigantism. While bearing no resemblance to an overweight person he nevertheless weighted $199 \mathrm{~kg}$ at his death, implying a BMI of 26.9 . It is possible that the standard rules of physiology do not apply to this case. Nevertheless, it seems reasonable to view his ultimately height as a proxy for the maximum height a human can attain. That is, as a proxy for $m_{\max }$. ${ }^{13}$ Note that this conclusion does not preclude (incidentially) wages that are suboptimally low, see Section 4 for a discussion of these cases.
} 
for effort given, the figure shows that PAL is slightly higher for heavier (larger) workers. The differences are, however, modest. Nevertheless it is worth stressing that similar "idiosyncratic" PAL differences across individuals are observed in experimental data (Westerterp, 2001). Hence, the model is consistent with available evidence in this respect as well.

In comparison to cross-individual variations in PAL the associated variation in energy needs is much larger, as documented in the left hand side panel of Figure 2. Mechanically this is a result of the fact that energy requirements vary with body mass in accordance with $m^{b+e c}$, whereas PAL varies only with $m^{e c}$. Quantitatively the model suggests that workers weighing 60 and 95 $\mathrm{kg}$ (the relevant size interval for practical purposes) require 1510 and 2045 calories per day to sustain their resting body (basal metabolism, $e=0$ ). At full effort, however, those requirements rise to 3260 and 4613 calories per day, respectively. Reassuringly, these calibrated requirements are well in accord with those suggested by FAO (2001) for similar body sizes and activity levels.

Mayer et al. (1956) show, for a sample of ca. $60 \mathrm{~kg}$ heavy West Bengali workers that as physical activity rises from light work (clerks and mechanics) to very heavy worker (e.g., blacksmith, carriers, and cutters) calorie intake per day rises by roughly the amount indicated by the solid line in Figure 2. This is consistent with the assumption that workers are being compensated for their exerted effort.

Figure 2: Effort and Calorie Consumption (left) - Effort and PAL (right)
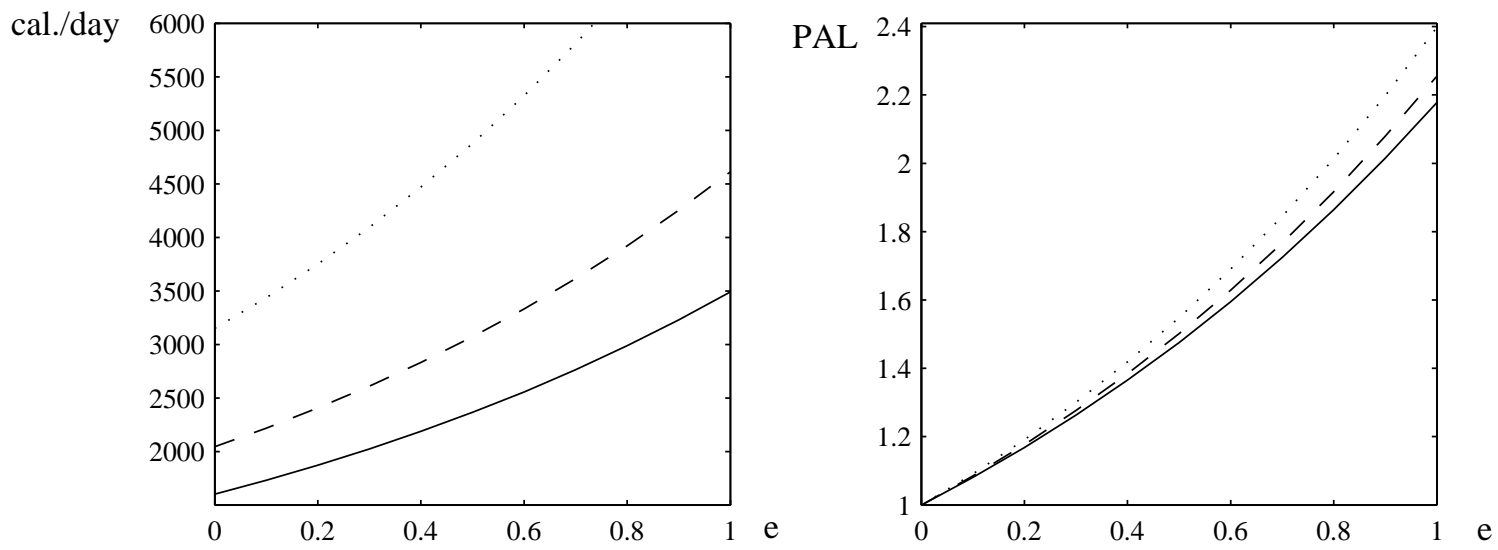

Parameters: $a=70 b=0.75, c=0.107$ and $\eta=1.39$. Results are for non-obese workers of $65 \mathrm{~kg}$ (solid lines), $90 \mathrm{~kg}$ (dashed lines) and $160 \mathrm{~kg}$ (dotted lines).

Based on these considerations we can now calculate the gap between equilibrium wages and the wage required for basal metabolism, or subsistence. This ratio is given by $w /\left(\epsilon a \cdot m^{b}\right)=\eta m^{c}$. 
As a result, the model motivates an upper boundary on the gap between the efficiency wage and subsistence which is $((2.4-1) \cdot 100=140 \%)$. This is the energy discrepancy at full working activity if the individual weights $160 \mathrm{~kg}$; our assumed upper boundary for body mass, associated with maximum PAL. At, for instance, a body weight of $65 \mathrm{~kg}$ the comparable number is $(2.2-1) \cdot 100=120 \%$ (see Figure 2). Hence, under plausible assumptions the model can generate wage level, which are consistent with those reported in Swamy (1997) and Dasgupta (1997). Since lowering the wage below the levels implied by the model would induce workers to reduce their effort $(e<1)$, producers would not be inclined to accept offers by unemployed workers who are willing to work for less than the going rate.

\section{An EXTENDED MODEL: HeTEROGENOUS BODY SIZE}

3.1. A re-parameterisation: focusing on height. In order to answer the question of who the unemployed are we have to introduce some heterogeneity of the labour force. The empirical literature supports a strong link between height and economic performance (Strauss and Thomas, 1998). We therefore proceed by investigating how biological fundamentals determine employment and wages for workers of different height by maintaining the assumption of individual body mass at equilibrium. In this manner, we extend our model to offer a biologically founded theory of the relationship between height and wages and between height and employment.

Accordingly we convert mass into height $h$ using that BMI $B=m / h^{2}$ where mass is measured in kilograms and height in meters. The efficiency wage (6) expressed in terms of height is

$$
w=B_{0} \cdot h^{2(b+c)}
$$

where $B_{0} \equiv(a \eta / \epsilon) \cdot B^{b+c}$. The model thus suggests a wage elasticity with respect to height of between 1.6 and 1.8. The re-parametrisation still allows us to match the data reasonably well, when it comes to the wage-height association.

One way to see that the elasticities are reasonable (though slightly on the low side) is the following. Compare two workers who are $160 \mathrm{~cm}$ tall and $161.6 \mathrm{~cm}$ tall, respectively. The latter is $1 \%$ taller than the former $(1.6 \mathrm{~cm})$, implying about $1.7 \%$ higher wages. According to the estimates of Schultz (2002) a height difference of $1.6 \mathrm{~cm}$ would imply a wage difference of between 2.24 and $2.72 \%$. 
Another way of gauging the relevance of the implied height-wage association is by comparison to the work of Strauss and Thomas (1998). The left hand side panel of Figure 3 shows the wage for height relationship for the benchmark calibration taken from Figure 2. For comparison with Strauss and Thomas (1998, Figure 2, which refers to Brazilian workers) we set the energy exchange rate $\epsilon$ to 1600. This implies for the calibration of $c=3 / 28$ and $\eta=1.39$ that a worker who is $170 \mathrm{~cm}$ tall, at a BMI of 22, gets a wage in log units of 0.75 . This number matches Strauss and Thomas' regression based estimate, at this height level. The full height-wage association is shown by the solid line. Dashed lines show wages when $c$ is allowed to increase to the maximum estimated value, i.e. $c=0.17$. If a different value for $c$ is employed, we need to recalibrate $\eta$. Following the same steps as above we obtain $\eta=1.01$. Visually comparing the figure with the data reported in Strauss and Thomas (1998) reveals that the slope of both curves underestimates the correlation between height and wage somewhat. ${ }^{14}$

The panel to the right demonstrates that the model also captures roughly the empirical association of BMI and wages as obtained by Strauss and Thomas (1998, Figure 3) for Brazilian workers. For that purpose we have held height constant at $1.60 \mathrm{~m}$ and evaluated (6) for alternative BMI. The body mass index, however, is mainly a measure of weight in disequilibrium, i.e. deviation from ideal weight, a problem that we will not address further. We henceforth assume that all workers display the same BMI (and $B_{0}$ is thus constant) but are of different height.

3.2. The influence of stature on production. The stature of workers is important for work performance since it is related to another physiological feature that we have not exploited so far: muscle force and human strength. Muscle power is proportional to muscle cross-section area which is measured in meters ${ }^{2}$ (Samaras, 2007). Since height is measured in meters, it follows that muscle force rises (scales) with height as $h^{2}$. This implies that if two workers of the same BMI, exerting the same (e.g. full) effort, the taller one contributes more force to the production process. ${ }^{15}$ Controlling for this feature and allowing for heterogeneity, the production function (3) should be rewritten as $Y=F\left(\sum_{i=1}^{L} e_{i}(w) \cdot h_{i}^{2}\right)$, where $e_{i}$ and $h_{i}$ are effort and height of the $i$-th worker employed, respectively.

\footnotetext{
${ }^{14}$ Actually, it would be worrisome if the present model could fully account for the causal effect of body size on labour productivity. In practice, height likely confers other productivity benefits beyond that which relates to 'brawn'. See, for example, Case and Paxson (2008).

${ }^{15}$ See Astrand and Rodahl (1970); Bale et al. (1994); Crewther et al. (2009); Peters (1983), and Zoeller et al. (2007).
} 
Figure 3: Height and Wages (left) - BMI and wages (right)
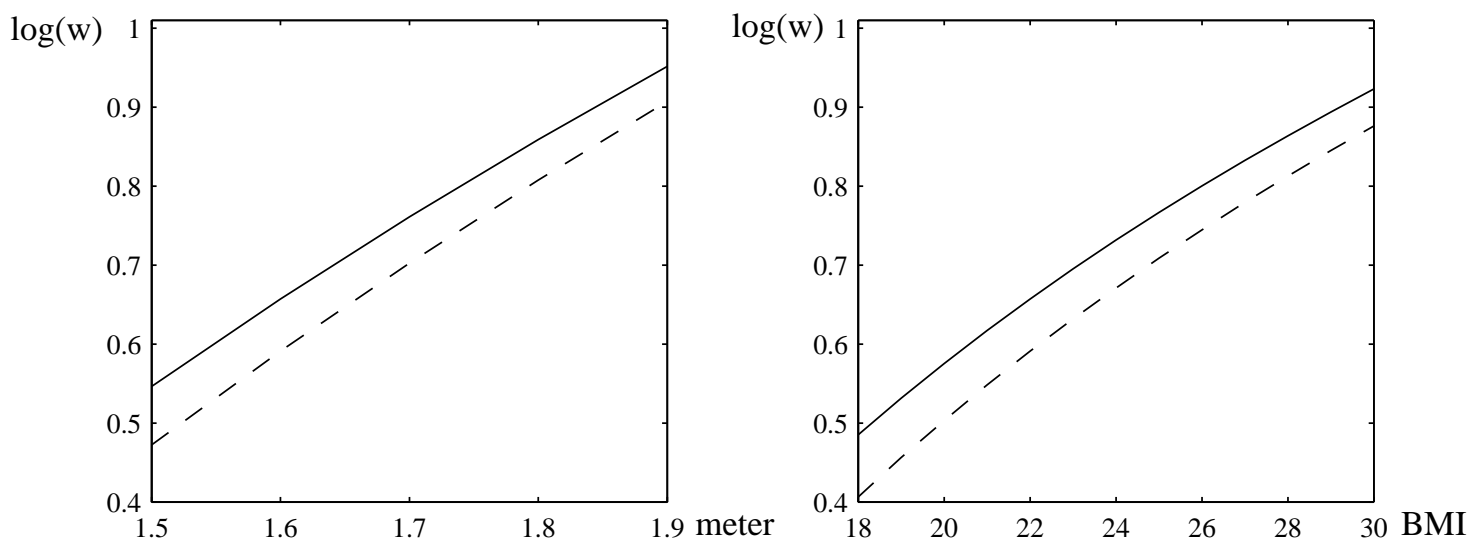

Parameters: $a=70, b=3 / 4, \epsilon=1600$ (both lines), $c=0.107, \eta=1.39$ (solid lines) and $c=0.17, \eta=1.01$ (dashed lines).

However, not all production processes rely on 'brute force' to the same extent. The literature in sport physiology differentiates between tasks that are mainly built on exertion of force (lifting weight, pushing, pulling), tasks of moving (running, jumping) and tasks of supporting body weight (sit-ups, push ups). Theoretical reasoning and empirical estimates suggest that individual performance in these tasks scales with height as $h^{\phi}$ where $\phi=2$ for exerting force, $\phi=0$ for moving and $\phi=-1 / 3$ for supporting body weight (Markovic and Jaric, 2004). ${ }^{16}$ These results are rather intuitive if one recalls and compares the visual appearances of Olympic medal winners in, say, the disciplines of rowing, running, and gymnastics. ${ }^{17}$

In wage work we expect tasks to be much more complex than those of the experiments in sports physiology. Still, there are undoubtedly tasks that rely to a great extent on exerting force. For example, force-intensive work like plowing and digging probably involves a height exponent of close to 2 . In contrast, working on an assembly line may be expected to be more or less unrelated to height, while carrying the mail in apartment buildings (lacking elevators) may put large individuals at a disadvantage. To capture this sort of heterogeneity across work tasks,

\footnotetext{
${ }^{16}$ Se also Bale et al. (1994) and Crewther et al. (2009) for further support that $\phi=2$ in force intensive tasks.

${ }^{17}$ Examining 800 adult Indian males, Singh and Singh (2007) find that blacksmiths and farmers on average are taller than tailors and carpenters. This is consistent with occupational selection taking place in accordance with the above considerations. Similarly, the French army (another force intensive occupation) introduced minimum height requirements in the 17th century, which later spread to other European armies (Komlos et al. (2003). In a similar vein, Kirby (1995) documents that coal miners were selected small so that they could fit the narrow coal seams, and Alter et al. (2004) document considerable height differences across occupations in 19th century Belgium; for instance, farmers were considerably taller than factory workers. See Steckel (1995) for a survey.
} 
we use a general exponent $\phi \in(-0.33,2)$, which one may think of as a weighted average of the three 'ideal' exponents, mentioned above.

To formalise these considerations, we assume a Cobb-Douglas production function, and measure labour as a continuous input factor:

$$
Y=A\left(\int_{0}^{L} e(i) h(i)^{\phi} \mathrm{d} i\right)^{\alpha} .
$$

The parameter $A$ controls for the general level of technology, and the parameter $\alpha, 0<\alpha<1$, controls for the ordinary productivity of labour with respect to the number of employed (in efficiency units).

3.3. Efficiency wages and the identity of unemployed. The efficiency wage of the last worker employed is $w(i)=B_{0} h(i)^{2(b+c)}$. The efficiency wage level is calculated by employing the Solow condition on the relevant effort function. The key thing to notice is that wages differ across workers with different sizes; bigger individuals are paid more.

Comparing exponents for productivity and costs of height (i.e., wages) we see that tall workers have an absolute advantage in being employed if $\phi>2(b+c)$, i.e. $\phi>1.7$ if $b+c=6 / 7$ as suggested by theory. Thus, if production is sufficiently force-intensive, taller workers are preferred. In this case we have the dual observation of bigger individuals receiving a higher wage, and being more likely to be employed. But if $\phi<2(b+c)$ smaller workers have an absolute advantage.

Accordingly, the model suggests that tall workers are preferred for heavy labour like unmechanised agriculture, construction work, and other relatively 'brawn-intensive' tasks. In contrast, small workers, including women and children, are preferred for assembly line production and other relatively fine-motor skill intensive activities. We discuss each case in turn.

Assume $\phi>2(b+c)$ and a work-force sorted in a descending order by height. Thus $h(L)$ is the height of the shortest 'just' employed worker. Since the worker is paid his or her efficiency wage and exercises maximum effort the first order condition for maximizing $Y-\int_{0}^{L} B_{0} h(i)^{2(b+c)} \mathrm{d} i$ with respect to employment size $L$ is

$$
\alpha A\left[\int_{0}^{L} h(i)^{\phi} \mathrm{d} i\right]^{\alpha-1} \cdot h(L)^{\phi}-B_{0} h(L)^{2(b+c)}=0 .
$$


Utilizing the substitution $\tilde{h}=h^{\phi}$ we get the following representation of the first order condition.

$$
\int_{0}^{L} \tilde{h}(i) \mathrm{d} i=\left(\frac{\alpha A}{B_{0}}\right)^{\frac{1}{1-\alpha}} \cdot \tilde{h}(L)^{\frac{\phi-2(b+c)}{(1-\alpha) \phi}} .
$$

Note that the re-scaling with respect to $\tilde{h}$ leaves the height ordering unaffected. Since $\tilde{h}(L)$ is monotonically declining in $L$ there exists a unique $L^{*}$ fulfilling (10). If the workforce exceeds $L^{*}$, unemployment exists and the shortest people are identified as being unemployed. The solution is visualised in the left hand side panel of Figure 4 where the falling curve represent the ordered distribution of height $\tilde{h}$ in the population and the integral below the curve up to $L$ identifies total employment. Hence, all workers taller than $\tilde{h}$ are employed and individuals below this threshold are unemployed. Note that this result is consistent with the observation of workers of different height performing identical tasks. For $\phi>2(b+c)$ our model thus provides a theoretical foundation for the observation that shorter people do not only earn less but are also less likely to be working (Strauss and Thomas, 1998).

The two other cases follow immediately. If $\phi<2(b+c)$ larger individuals will be rationed; in the particular case where $\phi=2(b+c)$ employers will be indifferent as to the height of their employees.

Observe that there is no incentive on the part of the employer to accept a low-wage offer from an unemployed. Employed workers are paid according to their metabolic needs, implying that any individual receiving less than that wage level would not exert full effort. In other words, if $\phi>2(b+c)$ there is no incentive for the employer to substitute a tall employee who needs (say) 4000 or more calories per day to perform his force-intensive work at full effort, by a smaller currently unemployed one that would need far less calories in order to sustain his metabolism in activity. As a result, 'undercutting' does not arise in equilibrium.

3.4. Comparative statics. As explained above; the link between body size and productivity depends, in this model, on the nature of the production process. That is, it depends on the nature of the tasks the individual workers perform. Technological progress, by changing the nature of the production process may therefore importantly affect the selection process, and the link between body size and employment.

The simplest comparative static exercise concerns a change in ' $\mathrm{A}$ ', which is neutral in the sense that it does not change the nature of the selection process. An increase in $A$ raises the marginal product of labour input (see the right hand side of equation (10)). As a result, if $\phi>2(b+c)$ 
, equilibrium height falls in order to re-establish equality. That is, employment rises, and so does the height of the shortest worker employed. In principle, our model inherits the shortcoming from the standard efficiency wage model that perpetual technological change eliminates unemployment. ${ }^{18}$ The same mechanism applies for an improvement of the diet captured by an increase of the energy exchange rate $\epsilon$. This lowers the wage costs per unit of body cell, $B_{0}$ decreases, and the multiplier on the right hand side of (10) increases implying an adjustment of $\tilde{h}(L)$ towards higher employment.

Figure 4: Height and Unemployment
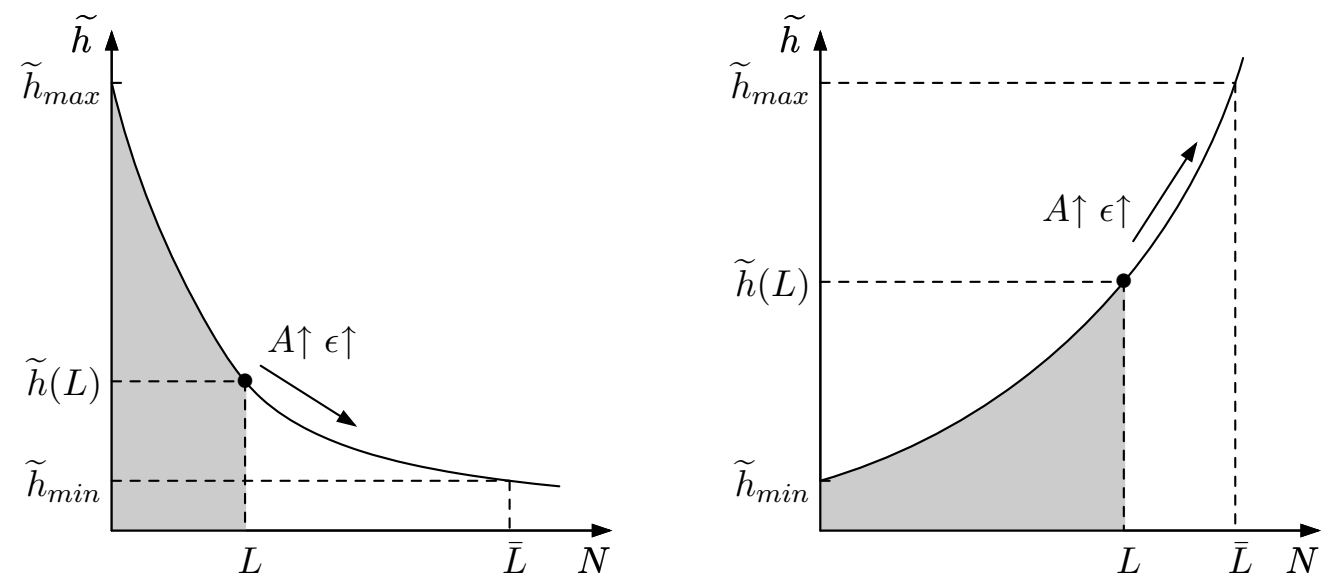

Left hand side: physiological advantage of tall people and unemployment (of size $\bar{L}-L)$ among short people for $\phi>2(b+c)$. Right hand side: physiological advantage of short people, unemployment among tall people for $\phi<2(b+c)$.

In contrast to the standard efficiency wage model we can also investigate task-specific technological progress. That is, technological change which influences the pay-off to body size (one could also label such innovations as 'height-biased).

Industrialisation, for example, replaces jobs in heavy agriculture (involving high $\phi$ 's) with assembly line jobs (with low $\phi$ 's around zero). At the moment where $\phi$ crosses $2(b+c)$ from above, firms prefer to employ smaller people because the contribution of an additional $\mathrm{cm}$ height on productivity falls below their marginal (efficiency) wage costs. Accordingly, previously unemployed small persons, like women and possibly children, increasingly enter employment as industrialisation proceeds.

\footnotetext{
${ }^{18}$ This shortcoming could, of course, be repaired as for the standard model by introducing psychological motives, e.g. the assumption that workers exert little effort when unemployment is low (Summers, 1988).
} 
This case is displayed in the right hand side panel of Figure 4. For that purpose we have ordered the population by height in ascending order and restated the equilibrium condition (10) as

$$
\int_{0}^{L} \tilde{h}(i) \mathrm{d} i=\frac{\left(\frac{\alpha A}{B_{0}}\right)^{\frac{1}{1-\alpha}}}{\tilde{h}(L)^{\frac{2(b+c)-\phi}{(1-\alpha) \phi}}} .
$$

The extended model predicts that taller persons are more likely to be unemployed. However, this tendency vanishes as technology (or the quality of diet) improves. In these cases the multiplier on the right hand side of $(11)$ increases and $\tilde{h}(L)$ has to get larger in order to re-establish equality. The model thus predicts that the association between height and unemployment should be less visible in technological advanced countries. ${ }^{19}$

\section{INEFFICIENT WAGES: FORCE INTENSITY AT WORK AND OBSERVABLE EFFORT}

In the analysis above we assumed that employers set wages in accordance with the Solow condition, which under plausible (biological) assumptions will induce the employees to exert full effort. Implicitly, of course, this solution requires employers to be able to observe the effort of the worker, which seems like a natural benchmark.

In some instances, however, it may be difficult to observe the workers effort fully. As argued by Foster and Rosenzweig (1994) the ensuing moral hazard problem may lead to wage differences across occupations where effort can be monitored to varying extent. For instance, whereas effort may be compensated fully in piece-rate work the same is not necessarily true for wage work rewarded on a time basis. As a consequence wages may be lower in the latter type of occupation than the former, reflecting the difference in the extent to which effort is observable to the employer.

To capture situations where effort is not fully observable in a simple way, suppose wages are set such that they are consistent with an effort level $\lambda, 0<\lambda<1$, that the employer expects from the employee. The required wage that would be consistent with effort level $\lambda$ is

$$
w(\lambda)=\frac{a}{\epsilon} \eta^{\lambda} m^{b+\lambda c}
$$

\footnotetext{
${ }^{19}$ There is evidence to suggest that height is also associated with improved cognitive abilities (Case and Paxson, 2008). If so, industrialisation may not work to lower $\phi$ insofar as the return to cognitive abilities rises during economic development. The basic mechanism explored above is, however, left unaffected.
} 
Naturally, this wage level is lower than $w^{*}$, the full effort wage. A relevant question is whether $w(\lambda)$ can be sustained. That is, does the fact that a currently employed worker exerts less than maximum effort allows undercutting to take place? The answer is found in the negative.

To see why, denote marginal profits for alternative effort levels, when employment is optimally adjusted, by $P^{\prime}\left(e, L^{*}\right)$. Next consider Figure 5, which depicts the $P^{\prime}\left(e, L^{*}\right)$ curve for varying levels of $e$. Evidently, undercutting will not take place as the wage, at $w(\lambda)$ is sub-optimally low. Indeed, workers receiving less than $w(\lambda)$ will exert even less effort than $\lambda$. As a consequence, the level of profits would deteriorate even further. Accordingly, the no undercutting result is robust against sub-optimally low wages associated with less than full effort.

Figure 5: Marginal Profits for Alternative Wage Rates

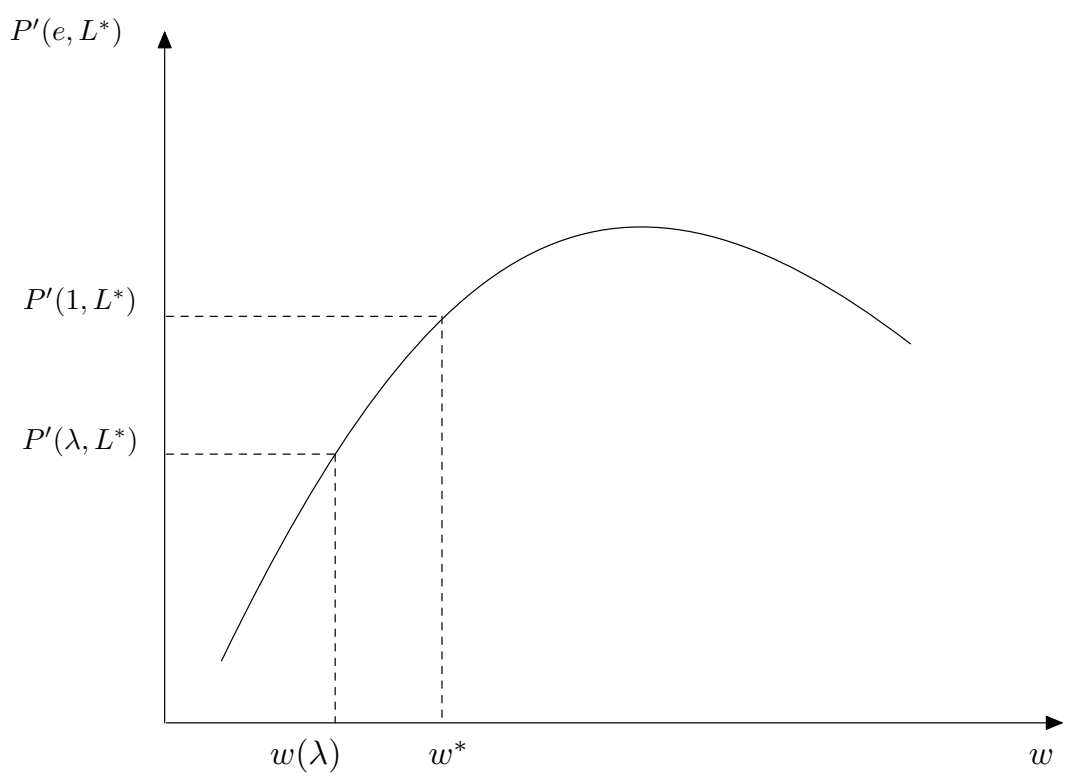

In essence, therefore, the results from Section 2 and 3 continue to hold: there is no basis for undercutting, and, controlling for stature one should not expect an association between wages and calorie intake. However, there is one important addendum. The absence of a link between wages and nutrition also requires one to take occupation into account. In particular, our theory predicts that workers in occupations where effort is more easily observable (e.g., piece-rate work) should obtain higher wages and consume more calories than workers in occupations where the monitoring problem is more severe. These predictions are borne out in the data (Foster and 
Rosenzweig, 1994). Moreover, the selection of workers into unemployment will also importantly be affected by the monitoring issue, as we show next.

Since equation (12) maintains the structure of the basic model we may proceed to study unemployment selection as we did in Section 3. First, observe that equation (12) can be restated in terms of height

$$
w=B_{0} h^{2(b+\lambda c)}
$$

with $B_{0}=a / \epsilon \eta^{\lambda} B^{b+\lambda c}$. Next, performing the same manipulations as in Section 3 we arrive at the conclusion that larger workers will be preferred in the labour market iff

$$
\phi>2(b+\lambda c) .
$$

The interesting new insight is that the condition is more likely to be met if the task at hand is force intensive and if effort is hard to observe. In other words, tall workers are particularly preferred in force-intensive work where effort is less easily observable; tree cutting or construction work, for instance.

This result has a straightforward intuition. Formally, $\lambda<1$ reduces the scaling exponent on body mass for an exercising organism and therefore makes larger workers, relatively speaking, even more efficient vis-a-vis smaller ones; with a smaller exponent the ratio of energy require-

ments to body size $\left(m^{b+\lambda c-1}\right)$ is declining faster in stature. Hence, pound-for-pound larger individuals are cheaper labour when effort is hard to observe, compared to the case where it can be observed easily.

This result suggests that micro econometric work on the determination of wages always should include information on occupation when examining the impact of body size on labour productivity. Since larger individuals are especially likely to be selected into activities where effort is hard to observe, a failure to take this selection effect into account may bias the coefficient on body size towards zero, as occupations where effort is hard to observe tend to carry lower wages.

\section{Conclusion}

The present paper has revisited the classical nutrition-based efficiency wage model. By providing physiological foundations for the nutritional requirements of effort, we demonstrate that the equilibrium wage should be considerably above subsistence, understood as energy requirements needed for basal metabolism. In addition, according to the model nutrition should not affect 
wages, conditional on body mass, in keeping with the evidence. An extension shows how the efficiency wage model can generate involuntary unemployment as well as provide (i) an explanation for the regularity that taller individuals tend to face a lower probability of being unemployed in less developed economies, and, (ii) provide an explanation for why taller workers simultaneously earn higher wages. Moreover, task-specific innovations may be one reason why a 'size bias' in unemployment may disappear during development. These results hold in spite of the fact that the biological founded effort function does not exhibit the convex-concave form postulated in previous contribution to the literature on the nutrition-based efficiency wage model.

A shortcoming of the analysis above is its partial equilibrium and static nature. Hence, an interesting topic for future work would be to provide a dynamic analysis, drawing on the basic framework above, in a general equilibrium setting.

Our conceptualisation of effort, as a measure of physical activity may allow for more precise tests of the nutrition based efficiency wage hypothesis. For example, one might be able to study the link between nutrition and physical activity levels of workers, and, in turn, physical activity levels on wages (in sectors where physical activity is key, of course). In this manner empirical research may move beyond "reduced form" regressions, when testing the nutritionbased efficiency wage model.

Acknowledgements. We would like to thank three anonymous referees, Stefan Klonner, Peter Svedberg and participants at the annual meeting of the Council for Developing Countries of the German Economic Association and the Nordic Conference in Development Economics 2008, as well as participants at several seminars for useful comments. 


\section{References}

Alter, G., Neven, M., and Oris, M. (2004) Stature in transition: a micro level study from nineteenth-century Belgium, Social Science History, 28, 231-47.

Astrand, P.O. and Rodahl, K. (1970) Body dimensions and muscular work, in P.O. Astrand and K. Rodahl (eds.) Textbook of Work Physiology, McGraw-Hill, New York, 321-39.

Banavar, J.R., Maritan, A. , and Rinaldo, A. (1999) Size and form in efficient transportation networks, Nature, 399, 130-32.

Bale, P., Colley, E., Mayhew, J.-L., Piper, F.C., and Ware, J.S. (1994) Anthropometric and somatotype variables related to strength in American football players, Journal of Sports Medicine and Physical Fitness, 34, 383-89.

Bliss, C. and Stern, N.H. (1978a) Productivity, wages, and nutrition: Part I: theory, Journal of Development Economics, 5, 331-62.

Bliss, C. and Stern, N.H. (1978b) Productivity, wages, and nutrition: Part II: some observations, Journal of Development Economics, 5, 363-98.

Bose, G. (1997) Nutritional efficiency wages: a policy framework, Journal of Development Economics, 54, 469-78.

Brody, S. (1945) Bioenergetics and Growth, Van Nostrand-Reinhold, New York.

Case, A. and Paxson, C. (2008) Stature and status: height, ability, and labor market outcomes, Journal of Political Economy 116, 499-532.

Crewther, B.T. , Gill, N., and Weatherby, R.P. and Lowe, T. (2009) A comparison of ratio and allometric scaling methods for normalizing power and strength in elite rugby union players, Journal of Sports Sciences, 27, 1575-80.

da Silva, J.K.L., Barbosa, L.A., and Silva, P.R. (2007) Unified theory of interspecific allometric scaling, Journal of Physics A, 40, F953-59.

Dalgaard, C.J. and Strulik, H. (2010) The physiological foundation of the wealth of nations. Discussion Paper 10-05 (University of Copenhagen). 
Darveau, C.A., Suarez, R.K., Andrews,R.D., and Hochachka, P.W. (2002) Allometric cascade as a unifying principle of body mass effects on metabolism, Nature, 417, 166-70.

Dasgupta, P. (1997) Nutritional status, the capacity for work, and poverty traps, Journal of Econometrics, 77, 5-37.

Dasgupta, P. and Ray, D. (1986) Inequality as a determinant of malnutrition and unemployment: theory. Economic Journal, 96, 1011-34.

Deolalikar, A. (1988) Nutrition and labor productivity in agriculture: estimates for rural south India, Review of Economics and Statistics, 70, 406-13.

FAO (2001) Human Energy Requirements, Food and Nutrition Technical Support Series 1, Rome.

Fogel, R.W. (1994) Economic growth, population theory, and physiology: the Bearing of longterm processes on the making of economic policy, American Economic Review, 84, 369-95.

Foster, A.D. and Rosenzweig, M.R. (1994) A test for moral hazard in the labor market: contractual arrangements, effort, and health, Review of Economics and Statistics, 76, 213-27.

Galor, O. and Weil, D.N. (1996) The gender gap, fertility, and growth, American Economic Review, 86, 374-87.

Heymsfield, S.B., Childers, D., Beetsch, J., Allison, D.B., and Pietrobelli, A. (2007) Body size and human energy requirements: reduced mass-specific resting energy expenditure in tall adults, Journal of Applied Physiology, 103, 1543-50.

Kirby, P. (1995) Causes of short stature among coal-mining children, 1823-1850, Economic History Review, 48, 687-99.

Kleiber, M. (1932) Body size and metabolism, Hilgardia, 6, 315-53.

Komlos, J. , Hau, M. and Bourguinat, N. (2003) An anthropometric history of earlymodern France, 1666-1766, European Review of Economic History, 7, 159-89.

Leibenstein, H. (1957) Economic Backwardness and Economic Growth: Studies in the Theory of Economic Development, Wiley, New York. 
Leonard, W.R. and Robertson, M.L. (1992) Nutritional requirements and human evolution: a bioenergetics model, American Journal of Human Biology, 4, 179-95.

Markovic, G., and Jaric, S. (2004) Movement performance and body size: the relationship for different group of tests, European Journal of Applied Physiology, 92, 139-49.

Mayer, J., Roy, P., and Mitra, K.P. (1956) Relation between caloric intake, body weight, and physical work, American Journal of Clinical Nutrition, 4, 169-75.

McFarlan, D. and McWhirter, N. (1991) The Guinness Book of World Records, Bantam, New York.

Mirrlees, J.A. (1976) A pure theory of underdeveloped economies, in L.G. Reynolds (ed.) Agriculture in Development Theory, Yale University Press, New Haven, CT.

Peters, R.H. (1983) The Ecological Implications of Body Size, Cambridge University Press, Cambridge.

Samaras, T.T. (2007) Human Body Size and the Laws of Scaling: Physiological, Performance, Growth, Longevity and Ecological Ramifications, Nova Science Publishers, New York.

Schultz, T.P. (2002) Wage gains associated with height as a form of health human capital, American Economic Review Papers and Proceedings, 92, 349-53.

Singh, A.P. and Singh, S.P. (2007) Impact of habitual physical activity on the human body proportions: a comparative study of some traditional occupational groups, Journal of Human Ecology, 22, 271-75.

Solow, R. (1979) Another possible source of wage stickiness, Journal of Macroeconomics, 1, 79-82.

Stiglitz, J. (1976) The efficiency wage hypothesis, surplus labor and the distribution of income in LDCs, Oxford Economic Papers, 28, 185-207.

Strauss, J. (1986) Does better nutrition raise farm productivity? Journal of Political Economy, 70, 297-320.

Strauss, J. and Thomas, D. (1998) Health, nutrition, and economic development, Journal of Economic Literature, 36, 766-817. 
Summers, L.H. (1988) Relative wages, efficiency wages, and relative unemployment, American Economic Review, 78, 383-88.

Swamy, A. (1997) A simple test of the nutrition-based efficiency wage model, Journal of Development Economics, 53, 85-98.

Svedberg, P. (2000) Poverty and Undernutrition, Oxford University Press, Oxford.

Steckel, R. (1995) Stature and the standard of living, Journal of Economic Literature, 33, 1903-40.

Weibel, E.R., Bacigalupe, L.D., Schmitt, B., Hoppeler, H. (2004) Allometric scaling of maximal metabolic rate in mammals: muscle aerobic capacity as determinant factor, Respiratory Physiology and Neurobiology, 140, 115-32.

Weibel, E.R. and Hoppeler, H. (2005) Exercise-induced maximal metabolic rate scales with muscle aerobic capacity, Journal of Experimental Biology, 208, 1635-44.

Weil, D. (2007) Accounting for the effect of health on economic growth, Quarterly Journal of Economics, 122, 1265-306.

West, G.B and Brown, J. H. (2005) The origin of allometric scaling laws in biology from genomes to ecosystems: towards a quantitative unifying theory of biological structure and organization, Journal of Experimental Biology, 208, 1575-92.

West G.B., Brown, J. H., and Enquist, B.J. (1997) A general model of the origin of allometric scaling laws in biology, Nature, 413, 628-31.

Westerterp, K.R. (2001) Pattern and intensity of physical activity, Nature, 410, 539.

Zoeller, R.F. , Ryan, E.D. and Gordish-Dressman, H. (2007) Allometric Scaling of Biceps Strength before and after Resistance Training in Men, Medicine 83 Science in Sports Ef Exercise, 39, 1013-19. 